\title{
A rib abnormality mimicking pulmonary nodule: a pitfall in the plain chest x-ray
}

\author{
Yeliz Akturk, Serra Ozbal Günes, Baki Hekimoglu \\ Department of Radiology, Diskapi Yildirim Beyazit Training and Research Hospital, Ankara, Turkey
}

\begin{abstract}
The ribs show a wide range of normal and pathologic radiographic appearences as well as congenital variations. Intrathoracic ribs are isolated and rare anomalies. They are usually super-numerary, more often right-sided, and involve the middle part of the thorax. We describe a case with intrathorasic rib abnormality mimicking a peripheral metastatic lung nodule in the plain chest x-ray and emphasize the use of coronal and sagittal reformatted images in thorasic imaging. Utilisation of multiplanar reformatted images in chest computerised tomography increase diagnostic quality.
\end{abstract}

\section{Introduction}

A wide variety of diseases affect the chest wall. Radiologists and clinicians have often difficulty in detecting, localizing, characterizing, and treating these disorders [1-3]. Costal abnormalities are frequently overlooked while evaluating the chest radiograms. Certain lesions in the ribs may be mistaken as pulmonary pathologies [4,5]. This can be important in patients with malignant diseases which may be primary or metastatic. The advent of cross-sectional imaging such as computed tomography (CT) and volume rendering techniques have enabled precise localization of chest wall lesions [1]. Here, we de-

Corresponding author: Yeliz Akturk, MD, Department of Radiology, Diskapi Yildirim Beyazit Training and Research Hospital, Irfan Bastug Street, Diskapi, 06120 Ankara, Turkey.

Tel.: +90.31.22596200 - Fax: +90.31 .23186690 .

E-mail: yelizakturk@yahoo.com

Key words: Rib abnormality; intrathoracic rib; pulmonary nodule.

Contributions: Yeliz Akturk: Diagnosis with CT and discussion; Serra Ozbal Gunes: Diagnosis with CT and discussion, Baki Hekimoglu: Supervision.

Conflict of interests: the authors declare that they have no conflict of interest to the publication of this article.

Received for publication: 27 February 2014

Accepted for publication: 4 July 2014

(C) Copyright Y. Akturk et al., 2015

Tipografia PI-ME Editrice, Italy

Monaldi Archives for Chest Disease Pulmonary Series 2015; 81:734

doi: 10.4081/monaldi.2015.734

This article is distributed under the terms of the Creative Commons Attribution Noncommercial License (by-nc 4.0) which permits any noncommercial use, distribution, and reproduction in any medium, provided the original author(s) and source are credited. scribe a case with intrathorasic rib abnormality mimicking a peripheral metastatic lung nodule in the plain chest $\mathrm{x}$-ray of a patient of breast carsinoma.

\section{Case report}

A patient with breast carsinoma was evaluated for detection of metastasis. An unclear nodular opacity in the right paramediastinal region on plain chest x-ray was detected in the initial evaluation (Figure 1). Suspecting a metastatic process, which may entirely alter the therapy plan, the patient was sent to chest CT for further evaluation. A high density sub-pleural nodular image was encountered in the right lung on routine axial images (Figure 2). On coronal reformatted images, two intrathoracic super-numerated ribs protruding to the lung parenchyma, which mimicked pulmonary metastatic nodular lesion, were spotted. Super-numerated ribs articulated with fourth and fifth thoracic vertebrae (Figure 3).

\section{Discussions and conclusions}

The ribs demonstrate a wide range of normal, congenital variant, and pathologic radiographic appearences [5]. These anomalies are rarely encountered in daily practice. These anomalies occur in $0.15 \%$ $0.31 \%$ of the population. Most of them are isolated anomalies, while some of them are related to syndromes. The findings pointing to abnormalities may be in the number, size, shape, or mineralization of the costae. These changes may be local or widespread. Normal variants are usually clinically insignificant, and often diagnosed during examination or incidental chest radiographs. However, since there are too many costal variants, whether the detected abnormality is pathologic or not should be clarified [4,6-8]. Certain lesions in the ribs may be mistaken as pulmonary pathologies $[4,5]$.

Intrathoracic ribs are very rare and isolated anomalies. Approximately forty cases have been reported so far, since it was described in 1947 for the fist time [7]. They are usually super-numerary, more often rightsided, and involve the middle of the thorax. The ribs protrude inwards from thorax cavity [4]. The development of this anomaly may be explained by both certain alterations in gene expressions and incomplete fusion of the sclerotome, from which the rib originate normally [7].

In our case there was an intrathoracic rib anomaly mimicking pulmonary metastasis in a female patient with breast cancer.

Several benign types of pathologic conditions can mimic the radiological appearance of neoplasm such as rib abnormalities. An indeterminate or incorrect diagnosis may lead to unnecessary patient anxiety, biopsy, and even surgery. The recent surge of imaging utilization, particularly in CT, has contributed to an increase in detection of such lesions or variations [9].

In our case, the rib anomaly was first spotted as an unclear opacity on a plain chest $\mathrm{x}$-ray. Since the patient had breast cancer, metastasis to the lungs was suspected. Therefore, the patient was sent to CT for evaluation of the lesion. On axial CT images, there was a high density 


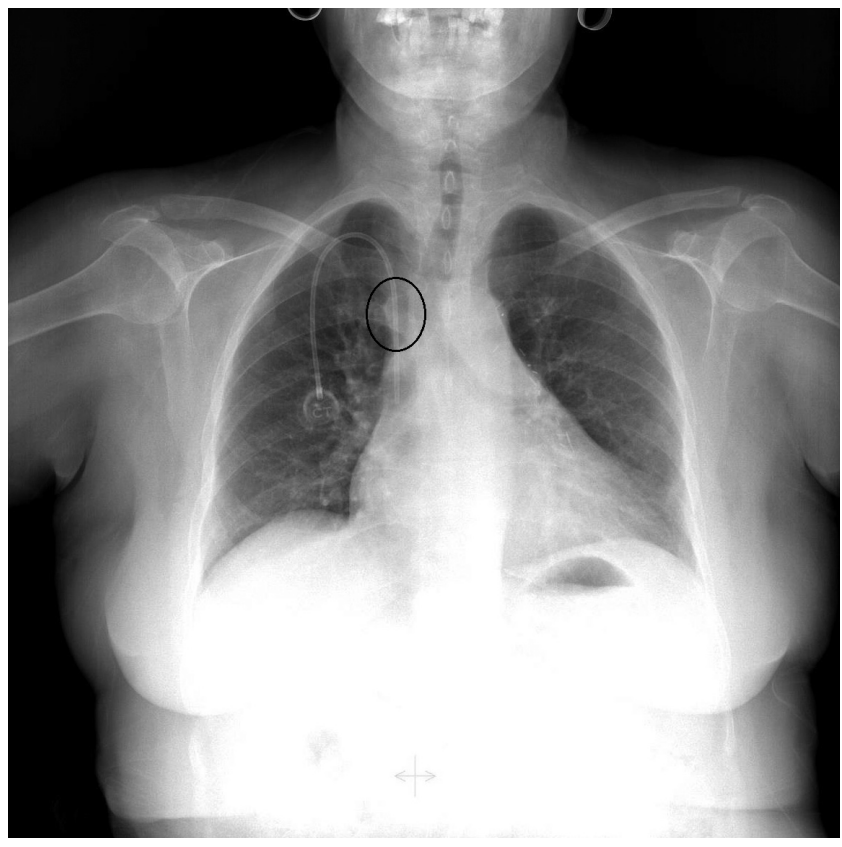

Figure 1. Plain chest $\mathrm{x}$-ray shows an unclear nodular opacity in the right lung. There is a port catheter in the vena cava superior.

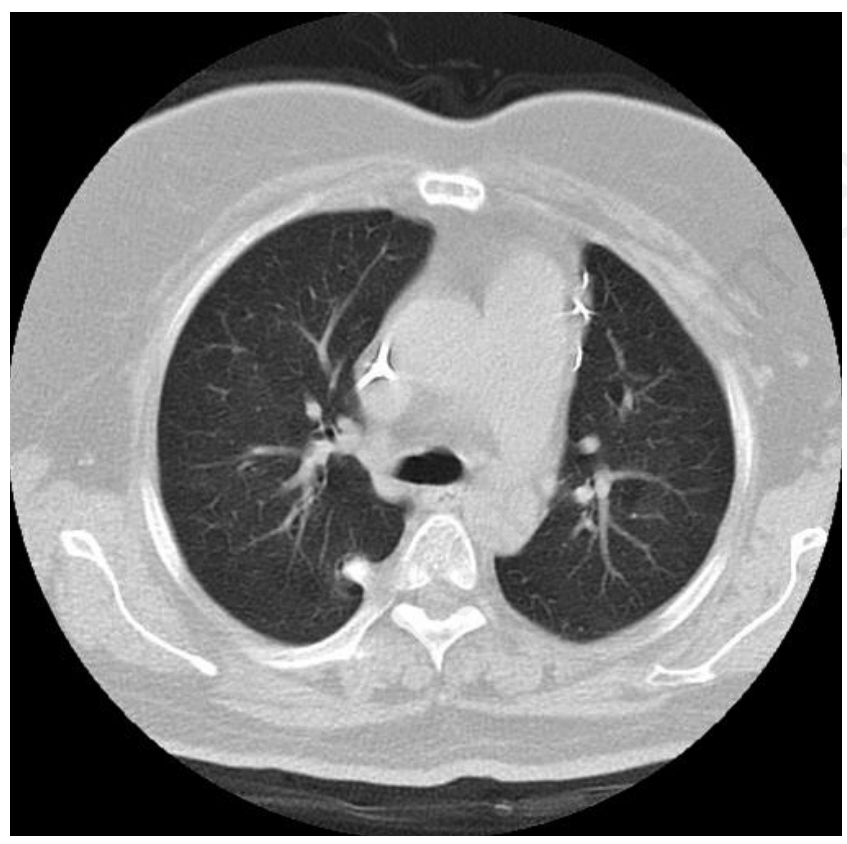

Figure 2. Axial image of computed tomography at the level of upper lobes, lung window demonstrates a high density sub-pleural nodular image in the right lung.

subpleural nodular image in the right lung which did not reveal a certain diagnosis. On coronal and sagittal multiplanar reconstruction of CT images two intrathoracic super-numerated ribs indenting to the lung parenchyma, which articulated with fourth and fifth thoracic vertebrae, were identified.

Several studies have shown that multiplanar reconstruction images can improve diagnostic accuracy when used in conjunction with axial

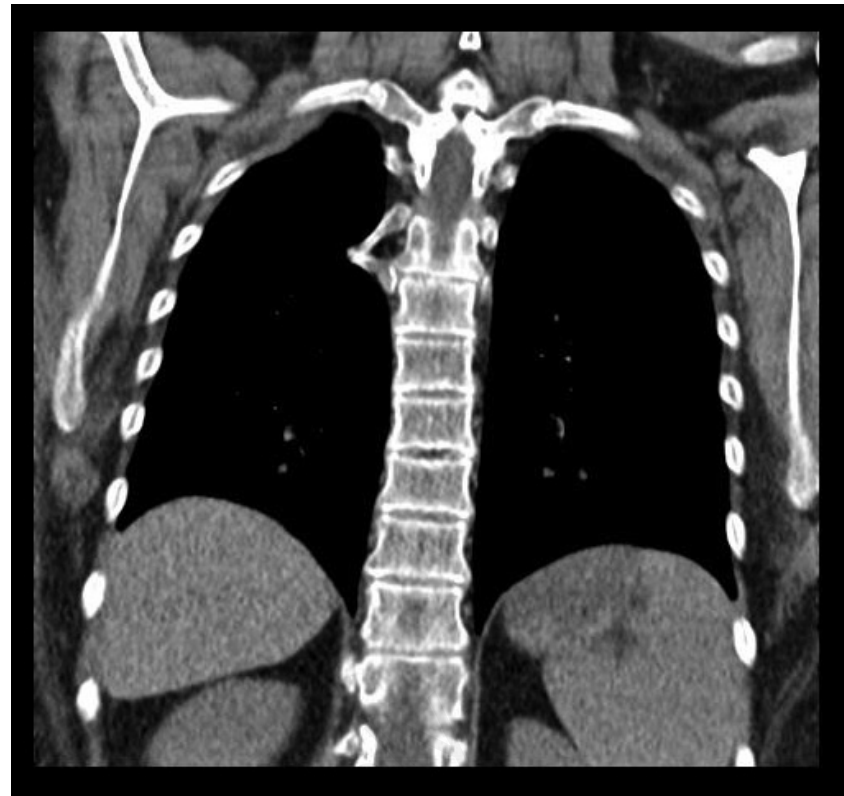

Figure 3. Coronal image of computed tomography shows two intrathoracic super-numerated ribs protruding to the lung parenchyma, which articulate with fourth and fifth thoracic vertebrae.

images for the assessment of certain chest abnormalities.With the advences in multi-detector helical CT (MDCT), utilisation of multiplanar reformatted images of the chest increased diagnostic quality [10].

Usually, although axial images may be adequate for diagnosis, volume rendered 3D images can provide more information about the nature of the disease and achieve better communication with clinicians. MDCT with 3D volume rendering has proved worthwhile in musculoskeletal imaging and can also be applied to imaging of the thoracic cage [11]. 3D imaging of bony structures is useful for evaluating fractures, dislocations and deformities of the chest wall. Volume scanning with MDCT assists in the identification of not only bony abnormalities but also soft tissue abnormalities [12].

As a result, the increasing use of MDCT and multiplanar reformatted images to establish or exclude a wide variety of diagnoses in many different clinical settings will likely increase recognition of non-neoplastic lesions that can simulate malignancies. Coronal and sagittal reconstructions have a major role in narrowing diagnostic considerations. In addition volume rendered images are often very useful for identifying costal abnormalities.

\section{References}

1. Jeung MY, Gangi A, Gasser B, et al. Imaging of chest wall disorders. Radiographics 1999;19:617-37.

2. Basara I, Balcı A, Koremez E. Diaphragm and chest wall pathologies. Available from: htp//www.toraks.org.tr. doi:10.5152/tcb.2013.26:140-156.

3. Tuncel M. Klinik radyoloji. İstanbul: Nobel\& Güneş Tıp Kitabevleri; 2008:345-53.

4. Argüder E, Akın A. Costal Abnormalities. Solunum 2012;14:6-13.

5. Guttentag AR, Salwen JK. Keep your eyes on the ribs: the spectrum of normal variants and diseases that involve the ribs. Radiographics 1999;19:1125-42.

6. Glass RB, Norton KI, Mitre SA, et al. Pediatric ribs: a spectrum of abnormalities. Radiographics 2002;22:87-104. 
7. Basarslan F, Bayarogulları H, Tutanc M, et al. Intrathoracic rib associated with pulmonary collaps in a pediatric patient. Iran J Radiol 2012:9;4:220-2.

8. Kurihara Y, Yakushiji YK, Matsumoto J, et al. The ribs: anatomic and radiologic considerations. Radiographics 1999;19:105-19.

9. Philips S, Restrepo CS, Chintapalli K. Mimics of neoplasia: Common lesions and findings misdiagnosed as malignancy. Applied Radiology 2012;2:5-14.
10. Kwan SW, Partik BL, Zinck SE, et al. Primary interpretation of thoracic MDCT images using coronal reformations. AJR Am J Roentgenol 2005;185:1500-8.

11. Lawler LP, Fishman EK. Multi-detector row CT of thoracic disease with emphasis on 3D volume rendering and CT angiography. Radiographics 2001;21:1257-73.

12. Ueno J, Murase T, Yoneda $\mathrm{K}$, et al. Three-dimensional imaging of thoracic disease with multi-detector row CT. J Med Invest 2004;51:163-70. 